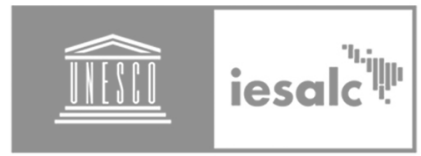

\title{
Unesco
}

Instituto Internacional

para la Educación Superior

en América Latina

y el Caribe
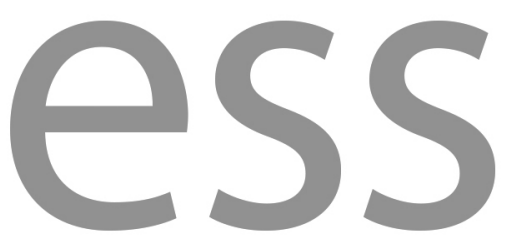

?

Educación

Superior y

Sociedad

Vol. 33 No. 2 (2021)

-

$\bullet$

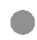

$\bullet$
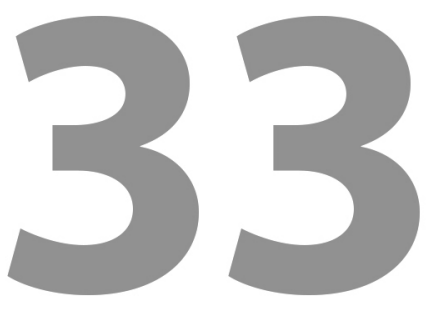

Incluye Dossier Temático:

Desafíos de la educación superior frente a la pandemia de Covid-19 en América Latina y el Caribe 


\section{La educación superior en el Perú en tiempos de pandemia y sus efectos en las trayectorias de estudiantes universitarios indígenas}

Inés Olivera, Alejandro Saldarriaga, María Amalia Pesantes

\section{RESUMEN}

El presente artículo busca ofrecer evidencias sobre los impactos de la COVID19 en la educación superior de jóvenes indígenas peruanos. Conociendo que se trata de una población históricamente excluida es muy importante señalar cómo la impactan de forma particular los escenarios de emergencia, tanto por la acumulación de los efectos de la exclusión histórica como por la renovada evidencia de acciones del Estado que siguen sin considerar las condiciones de vida de la población indígena. Basados en un ejercicio de estado del arte, al que se suman el seguimiento sincrónico de noticias, medidas oficiales, espacios virtuales (webinars) en donde se han expresado líderes y estudiantes universitarios, así como conversaciones personales con funcionarios, consideramos que aun con los límites que la pandemia impuso a la investigación cualitativa, este artículo tiene mucho para aportar al debate sobre el acceso, permanencia y egreso de los estudiantes de pueblos indígenas a la educación superior.

Palabras clave: Educación Superior; Estudiantes indígenas universitarios; Covid-19; Emergencia Sanitaria.

\section{Higher education in Peru in times of pandemic and its effects on in the trajectories of indigenous university students}

\section{ABSTRACT}

This research seeks to present evidence on the impact of COVID-19 on the higher education of Peruvian indigenous youth. It is highly important to point out how emergency scenarios especially impact these populations, taking into account that they have been historically excluded, not only because of the accumulation of the effects of historical exclusion, but also because of the renewed evidence of State actions that keep not taking into consideration indigenous 
peoples'life conditions. Based on an exercise related to the a state of art accompanied bysynchronous tracking of news, official measures, virtual spaces (webinars) where leaders and university students have expressed themselves, as well as conversations with officials, we consider that even with the limits that the pandemic has imposed in qualitative research, this article has much to contribute to the debate on access, permanence and graduation of indigenous students in higher education.

Keywords: Higher Education; Indigenous University Students; COVID-19; Health Emergency.

\section{Ensino superior no Peru em tempos de pandêmica e seus efeitos nos percursos universitários de estudantes indígenas}

\section{RESUMO}

O presente artigo tem como objetivo fornecer evidências sobre os impactos da Covid-19 no ensino superior dos jovens indígenas peruanos. Reconhecendoa como uma população historicamente excluída, é muito importante apontar quanto os cenários emergenciais a impactam de forma particular, tanto pelo acúmulo dos efeitos da exclusão histórica, quanto pela renovada evidência das ações do Estado que continuam desconsiderando as condições de vida da população indígena. A partir de um exercício sobre o estado do conhecimento, acompanhado de monitoramento sincrônico de notícias, medidas oficiais e espaços virtuais (webinars) onde líderes e estudantes universitários se expressaram, bem como conversas com funcionários, consideramos que mesmo com os limites que a pandemia impôs à pesquisa qualitativa, este artigo tem muito a contribuir para o debate sobre o acesso, permanência e graduação dos povos indígenas no ensino superior.

Palavras-chave: Ensino Superior; Universitários Indígenas; COVID-19; Emergência Sanitária. 


\section{L'enseignement supérieur au Pérou en temps de pandémie et ses effets sur les parcours universitaires des étudiants indigènes}

\section{RESUMÉ}

Cet article cherche à fournir des évidences sur les impacts du Covid-19 sur l'enseignement supérieur des jeunes indigènes péruviens. Prenant en considération qu'il s'agit d'une population historiquement exclue, il est très important de souligner comment les scénarios d'urgence affectent ces populations d'une manière particulière, à la fois en raison de l'accumulation des effets de leur exclusion historique mais aussi de l'évidence renouvelée des actions mises en place par l'État qui continuent à ne pas tenir compte de leurs conditions de vie. Sur la base d'un exercice d'état de la question accompagné du suivi synchrone de l'actualité, des mesures officielles et des espaces organisationnels indigènes où se sont exprimés dirigeants et étudiants universitaires, nous considérons que même avec les limites que la pandémie a imposées à la recherche qualitative, cet article a beaucoup à contribuer au débat sur l'accès, la permanence et l'obtention du diplôme des peuples indigènes à l'enseignement supérieur.

Mots clés : Enseignement Supérieur; Étudiants Universitaires Indigènes; Covid19; Urgence Sanitaire.

\section{INTRODUCCIÓN}

La pandemia por el COVID-19 ha afectado de manera desigual a diferentes grupos poblacionales en el Perú. Si bien diversas publicaciones han descrito y analizado el impacto de esta crisis en la salud de los miembros de los pueblos indígenas (Montag, 2021), poco se ha problematizado respecto del impacto de las medidas tomadas por el Estado peruano en materia educativa, específicamente, en estudiantes universitarios indígenas. La pandemia ha evidenciado que las insuficientes inversiones realizadas desde el siglo XIX en regiones rurales y territorios indígenas se traducen en un déficit en términos de acceso a servicios básicos como luz, agua, saneamiento, centros de salud, escuelas e internet por parte de las poblaciones indígenas Amazónicas y Andinas, según los datos presentados por el Instituto Nacional de Estadística e Informática (INEI) (INEI, 2016). 
Ante ello, esta investigación tiene la finalidad de describir lo que implicó la declaración del estado de emergencia en el Perú y los efectos que la pandemia por el COVID-19 tuvo en las trayectorias universitarias de estudiantes indígenas peruanos/as de la Amazonía, a partir de fuentes secundarias, usando la propuesta de Londoño, Maldonado y Calderón (2016) con relación a los trabajos exploratorios de tipo estado del arte. Así, esta propuesta constituye una primera recopilación documental, de carácter formativa e investigativa que gira en torno al reto de dar continuidad educativa durante el estado de emergencia a los y las estudiantes indígenas en universidades. En ese sentido este estudio representa un punto de partida para la formación de una postura crítica respecto de lo que se ha hecho y lo que no sobre un tema en específico (Londoño et al. 2016).

La información se ha recopilado con base en un trabajo de campo virtual realizado entre mayo de 2020 y enero del 2021, el cual ha acompañado sincrónicamente el devenir de la pandemia y del estado de emergencia en el Perú. Asimismo, se ha realizado un seguimiento de las modificaciones legales relacionadas con la inamovilidad obligatoria, las declaraciones del sector educación, de la superintendencia de educación superior, de las organizaciones indígenas y de los colectivos de estudiantes indígenas sobre los impactos de la emergencia sanitaria, los cuales serán complementados con una revisión crítica de material bibliográfico. A un año de la declaratoria del estado de emergencia por la crisis sanitaria, consideramos relevante hacer un balance de las medidas tomadas por el Estado peruano en términos de educación superior y su impacto en los y las estudiantes indígenas por dos razones fundamentales. Esta propuesta de investigación representa, por un lado, un aporte en tanto combina una serie de elementos que permitirán entender, de manera integral, el impacto de la crisis por el COVID-19 en el sector educativo de un país diverso en términos lingüísticos y culturales como el Perú. Por otro lado, este estudio permitirá formular nuevas preguntas y líneas de discusión en torno a lo que ocurre en las trayectorias universitarias de las y los estudiantes indígenas peruanos en el marco de la crisis sanitaria.

\subsection{La educación universitaria en el Perú y la subrepresentación de estudiantes indígenas}

Según la Ley General de Educación N²28044, publicada el 2003, la educación superior en el Perú está destinada a la investigación, creación y difusión de co- 
nocimientos, a la proyección a la comunidad y al logro de competencias profesionales de alto nivel, de acuerdo con la demanda y la necesidad del desarrollo sostenible del país. La educación superior no es obligatoria y puede ser impartida por entidades públicas o privadas, tales como institutos superiores o escuelas tecnológicas, artísticas o pedagógicas, o por universidades. Las universidades son orientadas por el Ministerio de Educación (Minedu) a través de la Dirección General de Educación Superior Universitaria y autorizadas a funcionar y supervisadas por la Superintendencia Nacional de Educación Superior Universitaria (Sunedu).

En el Perú, el proceso de organización y fiscalización de la calidad educativa en la educación superior es relativamente reciente. Es apenas en el año 2020, que se culmina la evaluación de la calidad de la oferta existente de educación superior universitaria mediante el proceso de licenciamiento de las universidades, el cual las habilita para funcionar. Este proceso se origina y es normado por la Ley Universitaria 30220, promulgada el año 2014, y por la Ley 29971, del 2012, la cual establece una moratoria que prohíbe la creación de nuevas universidades. Esta moratoria y su posterior ampliación (hasta abril del 2020), surgen para frenar el crecimiento excesivo y desordenado de universidades, la baja calidad del sistema universitario y el desgobierno de estas instituciones educativas, como consecuencia de las medidas implementadas durante la dictadura fujimorista de los años 90(1).

Al 4 de enero del 2021, según la web de la Sunedu, en el Perú existen 140 universidades, de las cuales 92 han obtenido licenciamiento. De estas, 46 son universidades públicas y 46 privadas. En el caso de las 49 universidades con licencia denegada, se trata de 45 privadas y dos públicas. Así, el reciente proceso de licenciamiento de universidades redujo a casi la mitad la oferta privada por no cumplir los criterios mínimos de calidad establecidos por dicha superintendencia.

\footnotetext{
(1) En el marco de la emergencia sanitaria, aunque no se ha ampliado formalmente dicha moratoria, la Sunedu no está trabajando en la creación de nuevas universidades y no hay registro de solicitudes de este tipo. Es importante señalar, también, que el 4 de junio del 2020 el congresista Gonzales Cruz elevó para el voto en el congreso el proyecto de ley para la ampliación por dos años más de la moratoria de creación de universidades; sin embargo, hasta la fecha, el proyecto no ha sido votado. Además, distintos congresistas han presentado en el congreso de la república proyectos para creaciones de universidades, los cuales no han sido aprobados por la comisión de educación del Congreso ni llevados a votación.
} 
Para incorporar a los estudiantes pertenecientes a universidades no licenciadas y asumir el aumento de la demanda, es que en febrero del 2021 se publicaron las Disposiciones para incrementar la oferta educativa de programas de pregrado en universidades públicas licenciadas (EI Peruano, 2021). Respecto de la presencia de estudiantes indígenas en el sistema universitario es importante señalar que la pertenencia étnica no es un criterio que las universidades o el sistema universitario suelan registrar. Para esto, las fuentes más recientes son la Encuesta Nacional de Hogares (Enaho) del 2015, el Censo Nacional del 2017 y el II Censo Nacional Universitario (2010). Según este último, para el año 2010 hubo 782.970 estudiantes de pregrado en el Perú, de los cuales el $97.3 \%$ aseguraron tener como lengua materna el castellano, $1.82 \%$ el quechua, $0.3 \%$ la lengua aimara y $0.6 \%$ "otras". Según Pérez-Campos y Espinoza-Lecca (2015), en el Perú existían, hasta el año 2010, 550 estudiantes cuya lengua materna es una lengua nativa amazónica. De estos, solo 178 son mujeres (32.3\%). Este documento, además, señala que la cantidad de estudiantes universitarios indígenas representa sólo el $0.07 \%$ del total de estudiantes universitarios del Perú, a pesar de representar el $0.6 \%$ de la población nacional de acuerdo al último censo del 2007, lo cual implica un claro problema de subrepresentación respecto de la cantidad total de estudiantes de educación superior.

Según los datos presentados por el INEI (2018) (2), a nivel nacional, en el 2015, un $28,9 \%$ de la población nacional de 15 años o más contaba con educación superior; y en el 2017, este porcentaje ascendió a 34\%. Sin embargo, si analizamos el porcentaje de personas con estudios superiores en función a su pertenencia étnica, vemos que de las personas que se autoidentifican como indígenas $u$ originarias de la Amazonía peruana, un 6.3\% declara tener estudios de educación superior universitaria, en comparación con aquellos que se autoidentificaron como blanca(o)/mestiza(o)/otra(o), entre quienes el alcance de la educación superior universitaria es de $22,1 \%$ (ob. cit.). Finalmente, de acuerdo a estos mismos datos, del total de la población censada al 2017 que asegura tener estudios superiores universitarios, solo el $0.3 \%$ corresponde a personas que se autoidentifica como indígena u originaria de la Amazonía ${ }^{(3)}$. Todo esto indica que la presencia

(2) Con base en la Encuesta Demográfica y de Salud Familiar del 2015 y la Encuesta Nacional de Hogares del 2015.

(3) La población censada incluida en la categoría "superior universitaria" incluye superior universitaria completa, incompleta y maestría/doctorado. 
de estudiantes indígenas amazónicos es muy limitada y que al mismo tiempo es una población que se encuentra por debajo de los promedios nacionales respecto de logros educativos.

\subsection{Acceso de estudiantes indígenas a la educación superior}

En el Perú, como en otros países de la región, la presencia de los y las estudiantes indígenas en las universidades es reciente y deficiente (Espinosa, 2017). Deficiente tanto en términos de su representatividad en la población universitaria nacional, como en términos de la adaptación al sistema universitario de estos/as nuevos/as actores/as universitarios/as. El sistema ha sido y es deficiente no solo para identificar y responder a las necesidades de estudiantes indígenas sino, sobre todo, para reconocer $y$ aprovechar sus fortalezas como aportes a sus procesos formativos y a las universidades. La acción explícita para promover el acceso de estudiantes indígenas a la universidad en el Perú data de la década de 1990, lo que es bastante reciente, más aún en un país de tradición universitaria centenaria. Sin embargo, para los pueblos indígenas, su visibilización como ciudadanos y el consecuente derecho a acceder a educación formal, y más aún, pertinente, es en general un proceso reciente y no del todo consolidado ${ }^{(4)}$.

Diversos estudios señalan que la educación superior no universitaria es la que congrega la mayor parte de estudiantes indígenas (Espinosa, 2007 y 2017; Ibáñez, 2014). Explica Espinosa (2007) que la opción por los institutos superiores frente a las universidades se debe a diversos aspectos: su mayor cercanía a los territorios indígenas, su menor costo en cuanto a derechos académicos, sus programas más cortos, menores dificultades de ingreso y permanencia por ser percibidas como de menor exigencia académica, la valoración de la formación para el trabajo, lo cual les permiten una pronta inserción laboral, etc. Dentro de estas opciones, un campo que además les posiciona con ventaja frente a los estudiantes no indígenas es el de la formación de maestros bilingües. De acuerdo a lo señalado en Pérez-Campos y Espinoza-Lecca (2015) respecto de los resultados del II Censo Nacional de Universidades 2010, de los 550 estudiantes universitarios indígenas peruanos, el 57,1\% escoge una carrera asociada a la pedagogía: 177

(4) Sobre la evolución y uso del concepto de indígena en censos peruanos, ver Valdivia (2011). 
siguen un programa para profesores de educación básica y secundaria, 102 un programa de profesores y/o maestros de primaria, y 35 uno de profesores de educación inicial y preescolar. Asimismo, 14\% escogen carreras relacionadas a agronomía, un $4 \%$ a derecho y a $24.9 \%$ otras.

Sin embargo, en este campo, la oferta educativa es también restringida y sus orígenes se encuentran en iniciativas de las organizaciones indígenas y de la iglesia católica, mas no del Estado. Desde las organizaciones indígenas, el caso más emblemático es el Programa de Formación de Maestros Bilingües de la Asociación Interétnica de Desarrollo de la Selva Peruana (Aidesep), iniciado en 1988 (Trapnell y Neyra, 2004). También desde Aidesep, se ha construido una oferta de educación superior tecnológica a través del Programa de Formación de Enfermeros Técnicos en Salud Intercultural Amazónica (PFETSIA) en el año 2005 (Rodríguez, Valdez y Reategui, 2009). Otra experiencia importante es la de la Federación de Comunidades Nativas de Madre de Dios (Fenamad), que en 1998 creó el programa de Educación Bilingüe Intercultural de Madre de Dios (Ebimad), en coordinación con el Instituto Superior Pedagógico Nuestra Señora del Rosario.

Respecto de acciones afirmativas para ampliar el acceso y permanencia de estudiantes indígenas a la educación superior, en el Perú lo que se ha tenido son acciones institucionales desde algunas universidades públicas. Algunos ejemplos emblemáticos son: la implementación de la Modalidad de Ingreso Aborígenes Amazónicos (MIAA)(5) por la Universidad Nacional Mayor de San Marcos entre los años 1998 y 2012, el programa de ingreso directo de estudiantes indígenas de la Universidad Nacional Educación Enrique Guzmán y Valle "La Cantuta" (Sanborn y Arrieta, 2011), o la modalidad de ingreso por pertenecer a un pueblo indígena de la región Loreto, implementada en la Universidad Nacional de la Amazonía Peruana (UNAP) el 2012, cuyo convenio incluye cuotas para ingreso, la exoneración del $100 \%$ del pago del examen de admisión, el pago del fondo estudiantil, la asignación de materiales de trabajo para cada especialidad y una cobertura al $69.24 \%$ del costo de la pensión alimenticia (Ibáñez, 2014). Sin embargo, como señala Espinosa (2017), es importante recalcar que todas estas iniciativas han surgido desde universidades específicas y no como política pública;

(5) Consultar, por ejemplo, Burga (2009); Villasante (2009); y Córdoba Cusihuamán (2012). 
además, los convenios son el resultado de la lucha y exigencia de organizaciones amazónicas, como Aidesep.

Como política pública para la promoción del acceso y permanencia de indígenas a la educación superior, lo que ha existido por parte del Estado es una tímida preocupación por el acceso de personas indígenas a este nivel. Se han creado dos modalidades en el programa Beca 18 específicamente destinados a la atención de jóvenes indígenas: la Beca Educación Intercultural Bilingüe, para formar maestros en Educación Intercultural Bilingüe (EIB), y la modalidad Comunidades Nativas Amazónicas (CNA), que define una categoría de postulación específica para jóvenes pertenecientes a comunidades nativas, a las carreras e instituciones de educación superior priorizadas por el programa Beca 18. Este programa nacional de becas, creado en el año 2011, está destinado a promover el acceso, permanencia y egreso de estudiantes indígenas en universidades e institutos de educación superior, priorizados por el sector en base a un ranking de calidad. Desde el Estado, además de becas, otra estrategia para ampliar la presencia de población indígena amazónica en la educación superior ha sido la creación de cuatro universidades interculturales(6), las cuales están dirigidas a atender a jóvenes indígenas, pero, como suele ocurrir con los servicios destinados a estas poblaciones, estas universidades cuentan con poco presupuesto y con condiciones difíciles de operación (Durand, 2017).

La insuficiencia de las medidas desplegadas para garantizar el derecho a la educación formal básica de los pueblos indígenas, que comenzó con la inclusión de las lenguas para la castellanización y que en los últimos años tendió a centralizar la interculturalidad en el discurso, se evidencia, en la brecha aún presente en el acceso a educación en todos los niveles de jóvenes indígenas frente a sus pares etarios en contextos urbanos.

En relación con la educación superior, son escasas tanto las medidas públicas como las iniciativas institucionales y privadas tomadas para garantizar el acceso y permanencia de estudiantes indígenas. Los programas institucionales univer-

\footnotetext{
(6) La Universidad Nacional Intercultural de la Amazonía (UNIA), creada en el 2000, comenzó a funcionar hacia el 2010 y fue la primera en obtener su licenciamiento. Entre 2010 y 2012 se crean otras tres universidades interculturales públicas: la Universidad Nacional Intercultural Fabiola Salazar Leguía y la Universidad Nacional Intercultural de la Selva Central Juan Santos Atahualpa, licenciadas el 2018; y la Universidad Nacional Intercultural de Quillabamba, licenciada el 2019.
} 
sitarios de ingreso especial y sus apoyos para la permanencia se implementaron casi en su mayoría en los primeros años del siglo xxı. Quedan muy pocos vigentes y se conoce poco sobre su funcionamiento e impacto. Considerando la brecha entre indígenas y no indígenas en términos de acceso a educación básica, lo reducido de la oferta de becas y de políticas de acción afirmativa para población indígena en educación superior, se evidencia una acumulación del incremento de la brecha por niveles. De esta forma, el panorama anterior a la pandemia, desde inicios de siglo hasta el 2020, muestra insuficientes esfuerzos y reducidos impactos en la garantía del derecho a la educación de los pueblos indígenas, principalmente en la superior universitaria.

\section{METODOLOGÍA}

\subsection{Análisis de las medidas desplegadas en el marco de la crisis sanitaria y su impacto en estudiantes indígenas}

Esta investigación busca describir los efectos de la pandemia y las medidas tomadas desde el Estado en las trayectorias universitarias de estudiantes indígenas en el Perú, a partir de la revisión de distintas fuentes como conversaciones personales con funcionarios, transcripción de webinars y revisión de normativas gubernamentales, las cuales serán analizadas en el siguiente orden: primero, se describirán las medidas referidas a la situación de universidades públicas y privadas implementadas por el Estado peruano en orden cronológico; y segundo, se recogerán algunos testimonios de estudiantes pertenecientes a poblaciones indígenas respecto del impacto de la pandemia en sus experiencias universitarias.

\section{RESULTADOS}

\subsection{Medidas implementadas por el gobierno}

El 12 de marzo de 2020 el Ministerio de Educación del Perú declara la suspensión de clases en todas las instituciones de educación superior hasta el 30 de ese mismo mes. Además de emitir disposiciones para la prevención, atención y mo- 
nitoreo del progreso del COVID-19, el Minedu señala la obligatoriedad de crear protocolos de acción en caso de aparición de casos de COVID-19 en instituciones educativas al retomar las clases presenciales, lo que, hasta ese momento, se esperaba que ocurriera el 1 de abril(7). Días después, se aprobaron los criterios para la supervisión de la adaptación de la educación no presencial, con carácter excepcional y temporal (8), pero es todavía con posterioridad, que se autoriza a las instituciones de educación superior a iniciar sus ciclos académicos en formato totalmente virtual, inicialmente contemplado como una medida temporal que daría paso a las clases presenciales desde el 4 de mayo(9).

El 18 de abril del 2020, a través del Decreto Legislativo № 1465 , se establecen medidas para garantizar la continuidad del servicio educativo en el marco de las acciones preventivas del gobierno ante el riesgo de propagación del COVID19 , las cuales autorizan a las instituciones educativas públicas, en todos los niveles, a adquirir y contratar dispositivos y servicios informáticos y/o electrónicos, así como la modificación, por parte de las universidades públicas y los gobiernos regionales, de los presupuestos aprobados para el año, lo cual evidenció haber tenido un efecto importante en tanto se logró que todas las universidades públicas (incluyendo seis aun no licenciadas) se encuentren funcionando de manera virtual. Como figura en el diario Gestión (2020), el director de la Dirección General de Educación Superior Universitaria (Digesu) del Minedu, señala que desde el inicio de la pandemia dicha dirección se ha concentrado en fortalecer las universidades públicas para garantizar la atención de sus estudiantes en cuatro líneas de acción: conectividad de los y las estudiantes e instituciones, capacitación docente, becas para estudiantes y desarrollo de políticas de bienestar. Todo esto permite a las universidades públicas avanzar en la virtualización e iniciar las clases, lo que se evidencia en el incremento de la oferta de educación superior universitaria pública desde el mes de mayo.

El 3 de mayo de 2020 se amplía, indefinidamente, la prohibición de inicio de clases presenciales ${ }^{(10)}$, lo que también impacta en el incremento del número

\footnotetext{
(7) Resolución Viceministerial 081-2020-MINEDU

(8) Resolución del Consejo Directivo № 039-2020-SUNED

(9) Resolución Viceministerial 084-2020-MINEDU

${ }^{(10)}$ Resolución Viceministerial Nº95-2020-MINEDU
} 
de universidades que inician el ciclo virtual. Y es frente a este escenario que comienza a evidenciarse la posible conclusión de uno o dos ciclos académicos en formato virtual. El 24 de agosto de 2020, la Sunedu publica las disposiciones para la prestación del servicio educativo superior universitario bajo las modalidades semipresencial y a distancia. En esta modificación se explicita que la prohibición de reanudar las clases virtuales está sujeta a lo señalado por la normativa vigente relacionada con la reactivación económica y las normas para la nueva convivencia social. De igual forma, se enfatiza en la continuidad del servicio educativo virtual contemplando la no vulneración del derecho a la educación de los y las estudiantes. Las modificaciones al presupuesto del sector educación permitieron que desde la Digesu y desde los presupuestos institucionales de las universidades públicas se destinaran fondos de apoyo a la conectividad de estudiantes y docentes, lo que resultó fundamental para la ejecución de la oferta educativa virtual.

Con base en la información declarada hasta mayo de 2020 en las páginas web institucionales, 59 universidades licenciadas (26 públicas y 33 privadas) ya habían iniciado las clases virtuales. Asimismo, para dicha fecha, otras ocho universidades públicas y tres privadas de las 92 licenciadas declaraban el inicio de clases virtuales entre finales de mayo y el mes de junio (Figallo et al., 2020). En una entrevista a TVPerú (28 de septiembre del 2020), el director de la Digesu, Jorge Mori, indicó que para septiembre todas las universidades públicas habían iniciado clases de manera remota.

Otras medidas importantes del sector en el marco de esta crisis han sido el incremento del presupuesto del Pronabec ${ }^{(11)}$. Desde la declaración de la emergencia sanitaria, Pronabec concentró sus esfuerzos en formular estrategias para la continuidad de los becarios ya insertos en los programas de becas y para continuar la convocatoria para nuevos grupos de becarios que comienza en agosto de

(11) Desde Pronabec ha habido un trabajo complejo por virtualizar los procesos para la continuidad del programa, así como del seguimiento de becarios/as. Esto ha requerido la creación de plataformas de información dentro de Pronabec para la presentación de documentos y la articulación con otras oficinas como la creación de la CLA (Constancia Logros de Aprendizaje), el SISFOH, la información sobre beneficiaros en la entrega de bonos por COVID-19 y otras instancias que permiten comprobar condición de pobreza (Núñez, comunicación personal, 14 de agosto del 2020). 
$2020^{(12)}$. En mayo del 2020, Pronabec lanzó las convocatorias de crédito y Beca para la Permanencia en la Educación Superior para ese año(13). Dicha beca se otorga a estudiantes de universidades públicas con muy buen desempeño académico y que, por su situación económica, no podrían continuar sus estudios. Así, para el 2020 se han entregado 8.470 becas, y en el contexto del COVID-19 se ha contemplado, también, la compra de laptops para dichos becarios, según lo señalado por Ana Núñez (comunicación personal, 14 de agosto de 2020), directora de la Oficina de Gestión de Becas del Pronabec. Es importante destacar que, según el portal web del Programa Nacional de Becas y Crédito Educativo (Pronabec, s.f.), aquellos postulantes que acrediten tener una condición económica de pobreza o pobreza extrema, haber sido víctima de la violencia entre los años 1980-2000, residir en distritos del VRAEM (Valle de los ríos Apurímac, Ene y Mantaro) o Huallaga, pertenecer a comunidades nativas, entre otros, recibirán puntuación adicional durante el proceso de selección de beneficiarios.

El 5 de octubre del 2020, desde su plataforma virtual, Pronabec (s.f.) anunció el inicio de la convocatoria del concurso Crédito Continuidad de Estudios 2020-II, dirigido a estudiantes de buen rendimiento académico afectados por la crisis del COVID-19. A esta beca pueden postular estudiantes no solo de universidades públicas o privadas licenciadas por la Sunedu, sino también de escuelas de educación e institutos tecnológicos y pedagógicos públicos y privados. La Beca Continuidad se otorga por un año, no hasta el fin de la carrera, aunque puede ser renovada(14) (Núñez, comunicación personal, 14 de agosto del 2020).

El 3 de diciembre del 2020 se crea la Beca Inclusión, una iniciativa de financiamiento a través de 100 becas, dirigida a estudiantes con discapacidad. Aquí, durante el proceso de selección, se otorga puntos adicionales a estudiantes mujeres,

${ }^{(12)}$ RDE N. ${ }^{\circ}$ 44-2020-MINEDU/VMGI-PRONABEC

${ }^{(13)}$ RDE N. ${ }^{\circ} 047-2020$

(14) Para la primera etapa, se anunciaron 10 mil becas; posteriormente, se anunció la ampliación a 14 mil y fueron asignadas 41 becas más. A la fecha el $70 \%$ de postulantes elegibles ya aceptaron la beca y ya están con resolución de becarios. Debido al éxito de esta primera convocatoria se ha planteado una segunda de 10 mil becas, anunciada la última semana de julio, y que, en principio, se ampliará para universidades públicas (Núñez, comunicación personal, 14 de agosto de 2020). 
a estudiantes que tengan condición de pobreza o pobreza extrema, a aquellos que residan en distritos del VRAEM o el ámbito del Huallaga, que pertenezcan a comunidades nativas amazónicas o campesinas, ser deportistas, u otras condiciones priorizables.

Tanto la Beca Permanencia como la de Continuidad de Estudios en Educación Superior consideran como población priorizable a jóvenes pertenecientes a comunidades nativas amazónicas, campesinas o poblaciones afroperuanas. Resulta importante señalar, además, que según información brindada por Pronabec a través de requerimientos ingresados por mesa de partes, el $20.18 \%$ de becarios de Beca Permanencia y el $4.01 \%$ de becarios de Beca de Continuidad de Estudios de la convocatoria 2020, pertenecen a una población indígena.

Adicionalmente, desde la gestión de becas, lo que se ha impulsado es agilizar la opción de suspensión de beca que existe en los contratos con los becarios. La opción de suspensión de beca permite a los becarios/as interrumpir por un ciclo o dos sus estudios y entregar sus becas, que podrán ser retomadas durante la reanudación de los estudios en el programa y universidad con las cuales fue beneficiario. Esta opción estaba contemplada para situaciones de enfermedad grave o de desastre natural que imposibilitaran la continuidad de estudios (Núñez, comunicación personal, 14 de agosto del 2020). Sin embargo, según información brindada por Pronabec (s.f.), sólo el $0.04 \%$ de becarios optó este año por la suspensión de la beca, siendo $2.3 \%$ el promedio en los tres años anteriores. En el caso de la modalidad Comunidades Nativas Amazónicas (CNA), si bien es la única que en el 2020 registró solicitudes de suspensión, éstas representan el $1.9 \%$ de becarios de la modalidad. Asimismo, la mayoría de becarios optó por aceptar la beca, aun durante la pandemia, manteniendo los porcentajes de aceptación de los años anteriores, con un porcentaje de aceptación de beca de $98.6 \%$ y $100 \%$ en la modalidad CNA este año.

Según un informe presentado por el Ministerio de Educación descrito en Alayo (2020), para septiembre del 2020 (a seis meses del inicio de la cuarentena obligatoria y el comienzo progresivo de las clases universitarias bajo la modalidad online) 174.000 jóvenes universitarios abandonaron sus estudios. Así, la tasa de deserción universitaria fue de $18.6 \%$ a septiembre del 2020 , seis puntos porcentuales más en comparación al $12 \%$ registrado en el 2019 . Asimismo, en las universidades públicas, la tasa de deserción asciende a 9.85\%, mientras que en las privadas a $22.5 \%$. Es importante destacar que, como ya se mencionó, en el 
marco de la emergencia sanitaria, distintas acciones fueron desplegadas por el Ministerio de Educación para apoyar a las universidades públicas y sus estudiantes, como la entrega de módems a estudiantes para ampliar la conectividad, la ampliación del número de becas permanencia asignadas a estudiantes de universidades y la creación de la Beca Continuidad. Esto, sumado a los costos de derechos académicos que tienen las universidades públicas, puede explicar que la tasa de deserción sea más del doble en universidades privadas que en las públicas. No obstante, cabría preguntarse cuál ha sido el impacto de la pandemia y de las medidas referidas a educación superior que han sido implementadas de forma progresiva, y específicamente, en los y las estudiantes pertenecientes a comunidades indígenas, por ser, con antecedencia, los más excluidos del sistema.

Sectorial e institucionalmente, la situación es profundamente compleja: los retiros de estudiantes han dejado a muchas instituciones educativas privadas con problemas de ingresos que, a su vez, han impactado en sus posibilidades de contratación, afectando el empleo. Así, algunas universidades han debido recurrir a la suspensión perfecta ${ }^{(15)}$ de algunos de sus empleados o a la disminución temporal de los haberes. La fiscalización y control de la calidad educativa también se ha complejizado, a pesar de la declaración temprana del sector respecto de sus preocupaciones y de sus esfuerzos.

Así, las medidas implementadas por el gobierno y las universidades para garantizar el servicio educativo en el año 2020 han sido la virtualidad y, sobre esta, se han establecido criterios para garantizar su control y calidad, además de haber establecido apoyos económicos para incrementar la conectividad de estudiantes y apoyar a los más golpeados por la crisis económica. Sin embargo, a lo largo del año lo que fue evidente, es que esta salida inevitable impactó particularmente en jóvenes indígenas, pertenecientes a sectores de mayor pobreza y con menor acceso a internet y equipos tecnológicos.

Para el año 2021 podemos avizorar un escenario similar. Según el Ministro de Educación, Ricardo Cuenca, se tiene previsto continuar con las clases bajo la

(15) La Suspensión Perfecta de Labores (Decreto Supremo N. ․ 011-2020-TR) fue una medida implementada por el gobierno que habilita el cese temporal de las obligaciones del trabajador y la prestación de sus servicios, así como de la remuneración respectiva por parte de su empleador, sin que esto implique la extinción de su vínculo laboral. 
modalidad virtual y, luego del análisis de distintos indicadores epidemiológicos, proponer, a través de la modificación y actualización de la Resolución Viceministerial $N^{\circ} 085-2020-M I N E D U$, el regreso progresivo a las clases presenciales (La República, 2021). El retorno a la presencialidad se ha vuelto una demanda de estudiantes, familias y del mismo sector para intentar frenar la desigualdad que se ha incrementado enormemente entre los más pobres sin acceso garantizado a servicios como internet y los sectores medios, lo que se refleja en una mayor exclusión de sectores rurales y de la población indígena.

\subsection{Testimonios de estudiantes indígenas amazónicos}

Si bien en la emergencia sanitaria la principal preocupación y motivo de debate y denuncia de las organizaciones indígenas ha sido la atención de salud y el acceso a las medidas de apoyo económico desplegadas por el gobierno, los problemas referidos a estudiantes indígenas de educación superior han sido también tema de seminarios y webinars en los últimos meses. Es en función a lo señalado en estos espacios que se describe, a continuación, la situación vivida por estudiantes indígenas respecto de la educación superior en el escenario de pandemia.

Un primer aspecto es que, como señala Martín Pizango (16), dirigente estudiantil indígena, presidente de la Organización de Estudiantes de los Pueblos Indígenas de la Amazonía Peruana (Oepiap), si bien actualmente los y las estudiantes indígenas de educación superior están viviendo una situación de exclusión en el marco de la emergencia sanitaria, y no han recibido apoyos focalizados para atender sus necesidades, es importante señalar que los problemas y dificultades son anteriores y vienen de décadas de incumplimiento por parte del Estado de sus obligaciones con la población indígena.

La pandemia ha recrudecido los impactos de los procesos de exclusión vividos por décadas por los pueblos indígenas amazónicos. La falta de inversión y consecuente insuficiencia de acceso a servicios básicos, como electrificación, de in-

\footnotetext{
(16) Participación en seminarios virtuales “Jóvenes amazónicos frente al COVID19: ¿cuál es el futuro de la juventud indígena amazónica en tiempos de pandemia?" (https://www.youtube. com/watch?v=0Jbpe3ieR_0) y "Los estudiantes indígenas en las universidades del Perú: situación y desafíos en la pandemia" (https://web.facebook.com/352926998171015/videos/373341640368177)
} 
fraestructura educativa, casi inexistencia de escuelas secundarias en territorios de comunidades nativas, y de deficiencia en calidad y pertinencia de la oferta educativa ofrecida en territorios indígenas, tiene por correlato una subrepresentación de la población indígena en la población estudiantil universitaria nacional. Por ejemplo, según los resultados definitivos del III Censo de comunidades nativas 2017, de las 2.703 comunidades nativas censadas, solo el $2.9 \%$ cuenta con acceso a internet (INEI, 2018).

Al respecto, en el Seminario GAA-GAMSI "COVID-19 y Amazonía Indígena", Martin Pizango (Grupo de Antropología Amazónica GAA-PUCP, 2020) afirma que, si bien los programas consideran a la población indígena como priorizable, estos terminan por reproducir los sistemas de exclusión al no considerar la realidad de los pueblos indígenas. Por ejemplo, señala Pizango (Grupo de Antropología Amazónica GAA-PUCP, 2020) que la Beca Hijo de Docentes está destinada a hijos de docentes con nombramiento; sin embargo, la realidad es que los maestros indígenas en escuelas EIB son contratados, pero no nombrados. Por otro lado, la Beca Talento Escolar exige que los aplicantes hayan participado en olimpiadas escolares; sin embargo, muchos/as jóvenes indígenas que estudian en sus comunidades no logran cubrir estos requerimientos porque dichos eventos no son realizados en estas zonas. Finalmente, todos los demás procesos que les imponen fechas que no contemplan sus calendarios y que, además, requieren de canales de información y postulación virtual, tampoco contemplan la realidad de los pueblos indígenas, muchos sin acceso a internet ni electricidad.

A todo esto, hay que sumar que, como recuerda José Antonio Dumas, dirigente de la Federación de las Comunidades Nativas del río Madre de Dios (Fenamad), encargado del área de educación y cultura:

[...] históricamente la educación en la amazonia siempre ha sido olvidada. Antes de la pandemia, siempre la educación ha sido precaria en las comunidades. Entendiendo que, en la misma EBR, donde algunas comunidades solo tenemos inicial y primaria y los estudiantes cuando acaban la primera deben ir a la ciudad estudiar la secundaria. Ahí sufren bastante los jóvenes. Muchas veces los jóvenes y hasta los mismos docentes en las universidades y en las ciudades sufren discriminación. A eso hay que sumarle la nivelación académica. En la región de Madre de Dios tenemos 7 colegios se- 
cundarios en comunidades nativas. Y ahí los docentes no son suficientes y a veces se les contrata solo para que cumplan las horas, no son bilingües... la educación es mala (11/10/2020, https://web.facebook. com/352926998171015/videos/373341640368177).

Con esta evaluación coincide Pizango, quien durante su participación en un webinar del Colegio de Antropólogos - Región Centro (2020), señaló que, en las comunidades nativas, las escuelas son de un nivel bajo, y que "cuando llegamos a la ciudad para estudiar [educación superior] nos chocamos con la dificultad de nivelarnos. Algunos se regresan, algunos se quedan, pero es difícil". La deserción aparece entonces como un problema anterior a la pandemia para estudiantes indígenas en secundaria y educación superior.

Lo que pasa entonces es que el estudiante se va a la ciudad para estudiar y a veces deserta porque sin familia en la ciudad sin apoyo, sólo, se sienten mal, es difícil y se regresan. Sin orientación ni apoyo, prácticamente están en el abandono. Y el estudiante abandona y deja de estudiar. Regresa a su comunidad o es captado por otras personas para realizar otros trabajos o situaciones que no les va a ayudar en su formación personal. A eso están expuestos. Es así la realidad de los jóvenes estudiantes indígenas. En la región amazónica de nuestro país la educación siempre ha estado abandonada (José Antonio Dumas, 11/10/2020, https://web.facebook.com/352926998171015/videos/ 373341640368177).

A esta realidad se suma, también, el actual escenario de pandemia, donde se agudizan los límites de estas políticas cuando no son diseñadas desde el reconocimiento de las brechas previas en el acceso a educación superior que sufren los y las estudiantes indígenas. Sobre esto, Seleni Rojas, estudiante de Lingüística en la Pontificia Universidad Católica del Perú (PUCP), perteneciente al pueblo Shipibo-Conibo, señala:

Esta pandemia me ha hecho ver cuán vulnerables somos los indígenas. Cuando comenzó la pandemia, cuando el presidente dio a conocer que esta enfermedad estaba aumentando, dio a conocer 
que debíamos quedarnos en cuarentena. Salieron muchos bonos y beneficios, pero nunca ha dado a los indígenas, pasaron varias semanas hasta que el estado de acordó de los pueblos indígenas. Se acordó para los bonos, pero nosotros dónde quedamos, dónde quedamos los estudiantes indígenas. Siento que hasta ahora el Estado no ha hecho nada por nosotros, siendo nosotros estudiantes que vamos a ser el futuro del país. Muchas veces el Perú es multilingüe y pluricultural, pero eso solo lo vemos cuando tenemos que danzar o hablar (25/06/2020, https://www.youtube.com/watch?v=0Jbpe3ieR_0).

Pensar en el impacto que han tenido la pandemia y el estado de emergencia en el país en la continuidad educativa de estudiantes indígenas universitarios, evidencia que las principales salidas del gobierno (inamovilidad obligatoria, virtualización de la educación y otras actividades) han tenido un efecto específico en las poblaciones sin acceso a internet ni servicios en sus localidades de origen, sin garantías de soporte económico, social y afectivo en las ciudades.

La propia medida de inamovilidad y su postergación progresiva colocaron a los y las estudiantes indígenas que estaban esperando el inicio de sus clases en las ciudades donde estudian en una encrucijada: mantenerse en ellas con acceso a internet para poder estudiar, pero solos y sin posibilidades de trabajar o de recibir apoyos económicos de sus familias; o volver a sus lugares de origen, sin internet, dificultando sus posibilidades de continuidad de estudios y sin una estrategia pública para gestionar su retorno con seguridad. Es así como entre mayo y julio, se observan procesos de retornantes en todo el país. Con una política lenta y de insuficiente cobertura para atender el retorno seguro desde el Estado, se observaron procesos de decenas y cientos de personas caminando a sus lugares de origen durante días y hasta semanas. Se ha señalado también que estos procesos de retorno han sido uno de los principales factores de expansión del virus y contagio en la región amazónica del país.

Como señala Junior Metaki (Grupo de Antropología Amazónica GAA-PUCP, 2020), estudiante machiguenga de Educación de la Universidad Católica Sedes Sapientiae (sede Nopoki), para muchos estudiantes indígenas volver a sus comunidades implicó un riesgo de salud muy grande por el largo traslado que deberían emprender. Por este motivo se encuentran en ciudades lejanas a sus comunidades y sin recibir apoyo económico de sus familias ni poder autogestionarlo. 
En su caso, los estudiantes de Nopoki cuentan con el albergue, pero sabe de estudiantes de otras instituciones que, sin apoyo, han tenido que regresar a pesar de los riesgos. Sobre esto, Martín Pizango (Grupo de Antropología Amazónica GAA-PUCP, 2020), resalta que la mayoría de estudiantes indígenas debe trabajar y estudiar, o depender del apoyo económico de sus familias, ambas situaciones limitadas por el estado de emergencia, lo que los dejó sin posibilidad de continuar con sus estudios. Señala también Seleni Rojas (Grupo de Antropología Amazónica GAA-PUCP, 2020), que mantenerse en las ciudades se volvió insostenible económicamente durante la crisis; sin embargo, volver a las comunidades implicó no poder continuar con los estudios a distancia. En ese sentido, como señalan ambos, es una realidad que en las comunidades no existen condiciones de conectividad similares a las de sus pares en las ciudades.

Con el inicio de la pandemia, algunos estudiantes estaban aún en sus comunidades y ahí han permanecido, otros decidieron volver, y como resultado, de los 120 estudiantes de la UNAP afiliados a la Oepiap, 90 se encuentran en la ciudad de lquitos (Pizango, 2020). Asimismo, Pizango (Colegio de Antropólogos - Región Centro, 2020), explica que a pesar de las gestiones realizadas por Oepiap para solicitar apoyo del gobierno regional e inclusive de congresistas de la región, no se ha logrado una respuesta pública que permita atender las demandas de los/las estudiantes, ni de salud, porque han sufrido también contagios, ni de conectividad para garantizar sus estudios. Sobre esto, Pizango (Colegio de Antropólogos - Región Centro, 2020), señala que a través de la congresista Luz Milagros Cayguaray se presentó al Congreso de la República, el 20 de julio de 2020, el Proyecto de Ley 05804/2020-CR para modificar la Ley 29837, que crea el programa nacional de becas y crédito educativo; sin embargo, el proyecto no fue aprobado en la votación y no se ha dado explicación de los motivos.

\section{DISCUSIÓN Y CONCLUSIONES}

A lo largo de este manuscrito, hemos descrito las implicancias de la declaración del estado de emergencia en el Perú y los efectos que la pandemia por el COVID-19 tuvo en las trayectorias universitarias de estudiantes indígenas amazónicos en Perú. Para lograrlo, este estudio se centró en el análisis de dos aspectos fundamentales: las medidas implementadas desde el Estado en materia de edu- 
cación superior, y los testimonios de estudiantes indígenas respecto del impacto de la implementación de dichas medidas en sus experiencias universitarias. En ese sentido, se puede concluir lo siguiente:

La implementación de cualquier tipo de medidas en un país de gran diversidad étnica especialmente aquellas con carácter de urgencia dada la emergencia sanitaria desde el 2020 requiere considerar la manera en que estas medidas afectarán a las distintas poblaciones. Sin embargo, como lo hemos mostrado, no hubo medidas específicas para universitarios indígenas, invisibilizando sus necesidades particulares.

Una de las posibles razones de esta invisibilidad, es la ausencia de bases de datos recientes que recojan información sobre las condiciones de los y las estudiantes indígenas en la educación superior. Si bien se puede encontrar cierta información en el Censo de Comunidades Nativas del 2017, esta no ha sido tabulada de forma que en el marco de la pandemia permita proponer medidas adecuadas para los estudiantes indígenas, como: la cantidad de estudiantes universitarios indígenas respecto del total en el país, la cantidad de estudiantes indígenas por centro de estudios y especialidades, la cantidad de alumnos y alumnas con posibilidad de conexión a internet, etc. Asimismo, resulta fundamental uniformar el concepto de indígena para el análisis y clasificación de dichos datos, usando de preferencia la autoidentificación étnica como el principal criterio.

Desde los testimonios de estudiantes, se puede evidenciar una constante en sus percepciones respecto del Estado y el tratamiento que históricamente las comunidades indígenas han recibido. Para ellos/as, ha existido un olvido que se ve reflejado en la calidad no solo de la educación superior, sino de la educación básica regular, pues, muchas, veces las trayectorias de los/as estudiantes se ven marcadas por la necesidad de alejarse de sus comunidades para seguir sus estudios en otras regiones y, posteriormente, en el peor de los casos, la deserción de dichos estudiantes ante la falta de recursos para completar los programas que han elegido.

Tal como afirmó Seleni Rojas (Grupo de Antropología Amazónica GAA-PUCP, 2020), estudiante de la PUCP, la pandemia ha evidenciado los distintos grados de vulnerabilidad en los que se encuentran las comunidades indígenas. En ese sentido, esta percepción de vulnerabilidad y olvido por parte del Estado debería ser contrarrestada a través de la implementación de medidas efectivas que se 
enfoquen en los/as estudiantes indígenas y sus potencialidades, tomando en cuenta, a su vez, las particularidades y limitaciones de cada región. Si bien existen iniciativas de financiamiento que contienen vacantes destinadas a miembros de comunidades indígenas y/o amazónicas, los requerimientos que estas exigen para la obtención de las mismas son difícilmente posibles de cumplir por parte de los miembros de algunas comunidades (Martín Pizango, 2020). Es por ello que resulta fundamental que, desde el Estado y con un enfoque efectivamente intercultural, se promuevan iniciativas que respondan a las necesidades de cada región, entendiéndolas como ampliamente diversas no solo en términos culturales y lingüísticos, sino en función al acceso a servicios básicos con los que cuentan (o no).

Finalmente, consideramos que, desde la academia, tenemos la responsabilidad de promover iniciativas que busquen problematizar respecto de la diversidad que caracteriza a países como el Perú, pues, como se evidenció desde los testimonios de estudiantes universitarios, la crisis sanitaria por el COVID-19 desveló las consecuencias de la postergación histórica en la atención a la población indígena. De esta forma, el principal impacto en términos de educación ocurre en estas poblaciones cuando las salidas planeadas por Estado, como la virtualidad, no se condicen con las condiciones de conectividad previas. Así, el COVID-19 sigue afectando de forma particular a quienes ya venían siendo perjudicados por las inequidades y postergación en el cumplimiento de derechos.

\section{AGRADECIMIENTO}

Este trabajo se llevó a cabo gracias a la ayuda de una subvención otorgada por el Centro Internacional de Investigaciones para el Desarrollo, Ottawa, Canadá a través del Proyecto "Supporting Indigenous Women in Science, Technology, Engineering and Mathematics in Peru". Las opiniones aquí expresadas no representan necesariamente las del IDRC o las de la Junta de Gobernadores. 


\section{REFERENCIAS}

Alayo, F. (28 de septiembre del 2020). Unos 174.000 estudiantes peruanos dejaron la universidad en lo que va del 2020. El Comercio.

https://elcomercio.pe/lima/sucesos/unos-174000-estudiantes-peruanos-dejaron-la-universidaden-lo-que-va-del-2020-noticia/?ref=ecr

Aprueban la Norma Técnica denominada"Disposiciones para incrementar la oferta educativa de programas de pregrado en universidades públicas licenciadas". (2021, 27 de febrero). El Peruano.

https://busquedas.elperuano.pe/normaslegales/aprueban-la-norma-tecnica-denominada-disposiciones-para-inc-resolucion-vice-ministerial-n-053-2021-minedu-1931260-1/

Clases en universidades de Perú 2021: ¿cuándo inician y en qué modalidad? (26 de febrero del 2021). La República.

https://larepublica.pe/sociedad/2021/02/27/clases-en-universidades-de-peru-2021-cuando-inician-y-en-que-modalidad-seran-atmp/?ref=Ire

Concytec (s.f.). Plan nacional de CTI 2006-2021. https://portal.concytec.gob.pe/index.php/concytec/estrategias/41-plan-nacional-de-cti-2006$\underline{2021}$

Decreto legislativo 1465-2020, del 18 de abril, que establece medidas para garantizar la continuidad del servicio educativo en el marco de las acciones preventivas del gobierno ante el riesgo de propagación del covid19. El Peruano. Lima, 19 de abril del 2020.

https://busquedas.elperuano.pe/normaslegales/decreto-legislativo-que-establece-medidas-paragarantizar---decreto-legislativo-n-1465-1865631-1/

Dumas, José Antonio. Webinario Los estudiantes indígenas en las universidades del Perú: situación y desafíos en la pandemia. Organizado por Colegio de Antropólogos - región centro 11/10/2020 https://web.facebook.com/352926998171015/videos/373341640368177

Durand, A. (Ed.). (2017). Educación superior intercultural indígena en el Perúy América Latina: aproximaciones, experiencias y desafíos. ITACAB.

Espinosa, O. (2008). Para vivir mejor: los indígenas amazónicos y su acceso a la educación superior en Perú. Revista ISEES - Inclusión Social y Equidad en la Educación Superior, 2, pp. 85-116. 
Espinosa, O. (2017). Educación superior para indígenas de la Amazonía peruana: balance y desafíos. Anthropologica. Año XXXV, №39, 2017, pp. 99122. http://www.scielo.org.pe/scielo.php?script=sci arttext\&pid=\$0254-92122017000200005

Figallo, F., Gonzales, M.T. y Diestra, V. (2020). Perú: educación superior en el contexto de la pandemia por el COVID-19. Revista de Educación Superior en América Latina, ESAL 8 (julio-diciembre, 2020). Monográfico. pp. 2028.

Ibáñez, M. (2014). Juventud, educación superior y movimiento indígena en el Perú. Chirapaq.

Instituto Nacional de Estadística e Informática (2016). Perú: condiciones de vida de la población según origen étnico. INEI. https://www.inei.gob.pe/media/MenuRecursivo/publicaciones digitales/Est/Lib1387/libro.pdf

Instituto Nacional de Estadística e Informática (2018). Perú:perfil sociodemográfico. Informe Nacional. Censos Nacionales 2017: XII de Población, VII de Vivienda y III de Comunidades Indígenas. INEI. https://www.inei.gob.pe/media/MenuRecursivo/publicaciones digitales/Est/Lib1539/libro.pdf

Ley Nº 28044 (2003). Ley general de educación. https://ccec.edu.pe/files/pdf/ReglamentoLey-N-28044.pdf

Ley № 29971 (2012). Ley que establece la moratoria de creación de universidades públicas y privadas por un período de cinco años. Diario Oficial El Peruano. https://busquedas.elperuano.pe/normaslegales/ley-que-establece-la-moratoria-de-creacion-de-universidades-ley-n-29971-881881-3/

Ley Nº 30220 (2014). Ley universitaria. Ministerio de Educación. http://www.minedu.gob.pe/reforma-universitaria/pdf/ley universitaria.pdf

Londoño, O., Maldonado, F., y Calderón, L. (2016). Guía para construir estados del arte. International Corporation of Networks of Knowledge. https://iconk.org/docs/quiaea.pdf

Metaki, Junior en Webinario Jóvenes amazónicos frente al COVID19: ¿Cuál es el futuro de la juventud indígena amazónica en tiempos de pandemia? organizado por GAA-GAMSI 25/06/2020 https://www.youtube.com/watch?v=0Jbpe3ieR o

Minedu: Más de 174 mil estudiantes dejaron la universidad en lo que va del 2020 (28 de septiembre del 2020). TVPerú Noticias. https://tvperu.gob.pe/noticias/nacionales/minedu-mas-de-174-mil-estudiantes-dejaron-la-universidad-en-lo-que-va-del-2020 
Montag, D., Marco Barboza, Lizardo Cauper, Ivan Brehaut, Isaac Alva, Aoife Bennett, José Sanchez-Choy, Juan Pablo Sarmiento Barletti, Pilar Valenzuela, José Manuyama, Italo García Murayari, Miguel Guimaraes Vásquez, Celso Aguirre Panduro, Angela Giattino, Edwin Julio Palomino Cadenas, Rodrigo Lazo, Carlos A Delgado, Alfonso Nino, Elaine C. Flores, Maria Amalia Pesantes, Juan Pablo Murillo, Luisa Elvira Belaunde, Sergio Recuenco, Robert Chuquimbalqui, Carol Zavaleta-Cortijo1 (2021). Healthcare of indigenous amazonian peoples in response to COVID-19: marginality, discrimination and revaluation of ancestral knowledge in Ucayali, Peru. BMJ Glob Health. Jan; 6(1): e004479. http://dx.doi.org/10.1136/bmigh-2020-004479

Mori, Diana. Webinario Los estudiantes indígenas en las universidades del Perú: Situación y desafíos en la pandemia, organizado por Colegio de Antropólogos - región centro 11/10/2020 https://web.facebook.com/352926998171015/videos/373341640368177

Pérez-Campos, P. y Espinoza-Lecca, E. (2015). El universo amazónico: una mirada cuantitativa a los hechos y algunas conclusiones para políticas públicas. En: Evidencia para políticas públicas en educación superior; Vol. 1. Hans Contreras Pulache Editor. Programa Nacional de Becas y Crédito Educativo. Ministerio de Educación.

Pizango, Martín. Webinario Jóvenes amazónicos frente al COVID19: ¿cuál es el futuro de la juventud indígena amazónica en tiempos de pandemia? organizado por GAA-GAMSI 25/06/2020 https://www.youtube.com/watch?v=0Jbpe3ieR_0

Pizango, Martín. Webinario Los estudiantes indígenas en las universidades del Perú: situación y desafíos en la pandemia, organizado por Colegio de Antropólogos - región centro 11/10/2020 https://web.facebook.com/352926998171015/videos/373341640368177

Programa Nacional de Becas y Crédito Educativo (s.f.). Plataforma digital única del estado peruano. https://www.gob.pe/pronabec

Proyecto de Ley 05804/2020-CR (2020). Ley que modifica la Ley 29837 que crea el programa nacional de becas y crédito educativa. https://www2.congreso.gob.pe/Sicr/TraDocEstProc/CLProLey2016.nsf/Sicr/TraDocEstProc/CLProLey2016.nsf/Comisiones/4DD9CE94CBFDA9E3052585AC00014995?opendocument 
Redacción Gestión (30 de septiembre del 2020). Minedu: universidades iniciarían clases semipresenciales en primer trimestre del 2021. Gestión.

https://gestion.pe/economia/minedu-universidades-iniciarian-clases-semipresenciales-en-primertrimestre-del-2021-noticia/?ref=gesr

Rodríguez Torres, R.A., Valdez Felipe, M.I. y Reátegui Silva, J. (2009). Formación de enfermeros técnicos en salud intercultural: una experiencia de cooperación entre las organizaciones indígenas de la Amazonía peruana, el estado y un instituto tecnológico. Educación superior, colaboración intercultural y desarrollo sostenible/ buen vivir - Experiencias en América Latina. Mato (ed.). pp. 87-114. IESALC-UNESCO.

Rojas, Seleni en Webinario Jóvenes amazónicos frente al COVID19: ¿cuál es el futuro de la juventud indígena amazónica en tiempos de pandemia?, organizado por GAA-GAMSI 25/06/2020 https://www.youtube.com/watch?v=0Jbpe3ieR 0

Sanborn, C. y Arrieta, A. (2011). Universidad y acción afirmativa: balance y agenda pendiente. En: C. Sanborn (Ed.) La discriminación en el Perú: balance y desafíos. pp.227-266. Universidad del Pacífico.

Trapnell, L. y Neyra, E. (2004). Situación de la educación intercultural bilingüe en el Perú. Documento de trabajo. Banco Mundial, PROEIB, Andes. https://centroderecursos.cultura.pe/es/registrobibliografico/situaci\%C3\%B3n-de-la-educaci\%C3\%B3n-intercultural-biling\%C3\%BCe-en-el-per\%(3\%BA

Valdivia, N. (2011). El uso de categorías étnico/raciales en censos y encuestas en el Perú: balance y aportes para una discusión. Documento de Trabajo, 60. GRADE Group for the Analysis of Development. https://nbnresolving.org/urn:nbn:de:0168-ssoar-51307-5

Zambrano, Gustavo. Webinario Martes de justicia y derechos. Derechos humanos de pueblos indígenas y amazónicos frente al Covid-19, organizado por Facultad de derecho PUCP - Ministerio de Justicia. 14/07/2020 https://www.facebook.com/217285458308343/videos/1225110037843743 


\section{BIONOTAS}

Inés Olivera. Doctora en antropología por la Universidad Nacional Autónoma de México (UNAM), maestra en Antropología por la UNAM y en Ciencias de la Educación por la Universidad Federal de Santa Catarina - Brasil (UFSC). Bachillera y licenciada en antropología por la Pontificia Universidad Católica del Perú (PUCP).

Correo electrónico: inesolivera@gmail.com

https://orcid.org/0000-0002-2088-2333

Alejandro Saldarriaga. Estudiante del último ciclo de la especialidad de Ciencia Política y Gobierno de la Pontificia Universidad Católica del Perú, Bachiller en Lingüística de la Universidad Nacional Mayor de San Marcos, y docente de inglés como lengua extranjera.

Correo electrónico:a20165976@pucp.edu.pe

https://orcid.org/0000-0002-7454-7354

María Amalia Pesantes. Doctora en Antropología Médica, Magíster en Salud Pública y Desarrollo Internacional. Actualmente es Profesora Auxiliar del Departamento de Antropología de Dickinson College (USA) e Investigadora Asociada en el Centro de Excelencia en Enfermedades Crónicas de la Universidad Peruana Cayetano Heredia. Correo electrónico: maria.amalia.pesantes@gmail.com https://orcid.org/0000-0002-0649-3649 